\title{
Implementing of Electronic Medical Record in Pelvic Floor Ward: A Pilot Study
}

\author{
Zinat Ghanbari' ${ }^{1}$ Nasrin Changizi2 ${ }^{*}$, Seyyed Reza Mazhari³, Tahereh Eftekhar1 \\ ${ }^{1}$ Department of Female Pelvic Floor Dysfunction, Tehran University of Medical Sciences, Tehran, Iran \\ ${ }^{2}$ Maternal Fetal and Neonatal Research Center, Tehran University of Medical Sciences, Tehran, Iran \\ ${ }^{3}$ Genetics Department, Shahid Beheshti University of Medical Sciences, Tehran, Iran \\ Email: Drz-ghanbari@yahoo.com, nasrin g2000@yahoo.com, \\ s.reza.mazhari@gmail.com, eftekhar35@yahoo.com
}

Received 12 May 2015; accepted 9 June 2015; published 12 June 2015

Copyright (C) 2015 by authors and Scientific Research Publishing Inc.

This work is licensed under the Creative Commons Attribution International License (CC BY).

http://creativecommons.org/licenses/by/4.0/

(c) (i) Open Access

\section{Abstract}

The electronic medical record is an essential technology tool to improve the quality of care. In present study we reported on the design and feasibility of electronic medical records in Female Pelvic Floor Dysfunction Ward. Our main goal was documentation with the least possible missed data, evidence-based decision making, documented active patient follow up and increasing patient's satisfaction. The Electronic Registry System of Female Pelvic Floor Dysfunction (Vali e Asr Hospital, Tehran, Iran) was designed in mid 2014 and tested till March 2015. The software description was designed based on previous paper questionnaire used in this ward. The electronic questionnaires were filled in upon hospitalization and thereafter including follow ups. The questionnaire included 10 demographic and 15 main questions. A digital analog scale (1 - 10) in each part quantified the effects of problem on patient's quality of life and also the effects of interventions as well. Entered information in each step was available for those with defined access. Reporting design was dependent on the needed data. Our supervised data entry was a guarantee to the quality of information with the least errors. Access to all para clinical data made rapid and evidence-based decision making. Patient satisfaction was achieved because of unified approach. The most interesting part was access to evidence-based results and data to be used in research projects. This study showed that EMRs in Female Pelvic Floor Dysfunction Wards could provide valuable information, improve the quality of care and increase patient's satisfaction.

\section{Keywords}

Electronic Medical Record, Registration System, Female Pelvic Floor Dysfunction (FPFD)

\footnotetext{
${ }^{*}$ Corresponding author.
}

How to cite this paper: Ghanbari, Z., Changizi, N., Mazhari, S.R. and Eftekhar, T. (2015) Implementing of Electronic Medical Record in Pelvic Floor Ward: A Pilot Study. Open Journal of Obstetrics and Gynecology, 5, 319-323. 


\section{Introduction}

Electronic medical records (EMRs) contribute dramatically to the improvement of the quality and efficiency of care. Moreover, cost effectiveness of EMRs has long been proven in different clinical settings [1] [2]. The EMR is an essential technology tool for health care, modernizing the management of medical information and contributing to high-quality patient care and efficient management [1]. Moreover, available charts have been reported as benefits of an EMR system by other studies [3]. Electronic medical records support clinical activities and reduce errors [4].

Most countries in Europe and the USA are using EMRs to improve health care quality. On the contrary, most developing countries face with lack of robust health care infrastructure in the form of information and communications technology (ICT) [3].

A systematic review showed a positive patient satisfaction with the electronic medical/health record [5]. Another survey suggests that physicians and nurses prefer the use of an EMR [4]. Some surgical subspecialties like female pelvic floor dysfunction (FPFD) group also may stand to benefit from an Electronic Medical Record because of high frequent and chronic conditions such as pelvic organ prolapses, pelvic pain, and sexual dysfunction which have impact on quality of life (QOL) over the span of decades as well as needs for long term follow up. So a high quality registry system can serve a longitudinal data from a large number of surgeons practicing in institutions [6].

There is no doubt that poor coordination of health care has negative consequences for patients and increase medical costs. Health information system (HIT) technology has the potential to improve this coordination. In present pilot study we reported on the design and feasibility of Electronic medical records and discussed the potential for such technologies in Female Pelvic Floor Dysfunction Ward (Vali e Asr Hospital, Tehran, Iran). The main goal was documentation with the least possible missed data, evidence-based decision making, documented active patient follow up and increasing patient's satisfaction.

\section{Materials and Methods}

Female Pelvic Floor Department is a sub specialized group in Obstetrics/Gynecology ward of Vali e Asr Hospital; a tertiary referral center and also one of the teaching hospitals affiliated to Tehran University of Medical Sciences (Tehran, Iran). Female Pelvic Floor Department has 10 beds for inpatient care service. This department is the first and till now the only training center for Female Pelvic Floor Dysfunction Fellowship in Islamic Republic of Iran. It has 2 operation days with at least 10 operations in a week.

The Electronic Medical Registry System of Female Pelvic Floor Dysfunction Ward as a pioneer was inserted in 2014 and tested up to March 2015 in health information system of Vali e Asr Hospital (Tehran, Iran). Three Steps were included in this project:

- Provision of structured Clinical Documenting Tool.

- Determining the flow of information in Software.

- Creating a reporting system with Excel Output.

Previous paper registry system was mainly used for providing the basic Information, chief complaints, worst complaints (combination of Validated ICIQ, POPDI-20) and added Validated PSIQ-12 in this registry system. It included inpatient reception, including all previous and present medical and pelvic history, diagnosis and planned surgery for present hospitalization, intra operative complication, time period, type of anesthesia, and post operative complications (during hospitalization or after discharge from hospital).

An electronic questionnaire was prepared based on literature text. It was evaluated by experts.

The questionnaire included 10 demographic and 15 main questions. In case of No as a negative answerer; this part would have been closed and in case of Yes (positive answers), detailed review of these questions should have been answered. A digital analog scale (1 - 10) in each part quantified the effects of problem on quality of life.

Physical Examination Part included General Examination, specially abdominal exam, DTR evaluation, Stress urinary incontinency evaluation (stress and cough test, PVR), Bulbocavernous reflex, Anal Wink reflex evaluation, Pelvic Organ Prolapse Quantification and Staging, Rectal and perineal body evaluation and Bimanual Uterine examination.

Based on patient's complaints the related questionnaire would be opened and filled up in details. We avoided open text answers to provide quantitative data in the output sheets. For going to the next main question at least all previous main questions should have been answered; consequently missing data were the least possible. 
In lab tests any para clinic tests including 3 days fluid volume chart, basic Lab results, Urodynamics Study, MRI-Defecography or Defecography, ultrasound, Analmanometry were included. In case of their presence of any of these tests, the details would appear to be filled. We also used indicators to have quantitative data in our output reports for para clinical Data.

A Digital Analog Scales (1 - 10) was used for scaling each complaint; before and after intervention determining the effect of the problem on patient's Quality of Life (QOL) and patient's satisfaction. Post interventional questionnaire (to assess patient's satisfaction and post intervention QOL) was designed to be filled after 2 weeks, 4 weeks, 3 months, and 12 months. Pop q also was repeated 6 months and 1 year after operation in follow up visits. In order to avoid missed tracking of some patients in traditional program, an alarm program for active patients' follow up was designed. Patients could be followed by phone call for reappointing an office visit.

More over in follow up program, some questions related to beginning of new complaints, recurrence or continuation of the previous complaints were inserted.

Based on complications in post intervention period, we used a combination of validated questionnaires ICIQ, PSIQ-12, andPOPDI-20.

It should be emphasized that for providing quantitative data in output report, open text answers were avoided as much as possible.

The Software description was designed by an information technology (IT) specialist in Restful Web services, J2 environment. Its data base was postgre SQL19.0 with prime faces JSF2.0 for user side framework and ORM (object relational mapping): Hibernate 4.0.

In servers, it could be installed on Linux, Windows 2003+, and in Browsers, on Mozilla Firefox $3.5+$ as well.

Our study was approved by the ethic committee of Tehran University of Medical Sciences.

\section{Results}

Our results showed that piloting of the system was a clue of the benefits achievable by running this system. Entered information in each step was available for those with defined access wherever they were. This opportunity made supervision of what was going on in the hospital, easier even from home.

Reporting design was dependent on the needed data (based on therapeutic approach, Surgeons, complications, Material used) and it could have been rapidly prepared. In addition on demand report extraction or periodic report extraction was feasible.

This supervised Data entry could guarantee the quality of information, with least of errors or neglects.

Access to all para clinical data caused rapid and evidence-based decision making. For each patient with a hospital reception number follow up in long term was possible, either via visiting in our Clinic, or via phone call, ...

Patient satisfaction was achieved because of unified approach and least of mistakes. In follow up visits confirmation of recurrence or continuation of the chief Complaint was possible.

With having Evident Output results for each problem or any intervention during hospitalization, we could use the results either in research programs or for redirecting some approaches that seems to be problematic.

\section{Discussion}

The goal of this study was to move towards an integrated multimedia registration and detailed information collection. Our Hospital Female Pelvic Registry System will be accomplished in near future with inclusion of ambulatory patients.

This study had demonstrated the successful development of an EMR for Female Pelvic Floor Department, as well as the ability to use the registry system to retrieve all structured and categorized data. In consistence to our results Goldberg et al. indicated that the technology has potential as a powerful tool in enhancing registry system [6]. Sujansky also pointed to a number of studies that have shown clinical event-monitoring systems can be effective in improving patient care in hospital settings; An Electronic Medical Record can provide comprehensive, organized and structured clinical data of various kinds, such as vital signs, laboratory results, radiology reports, current medications, problem lists, and past medical histories. These data generate a diagnostic decisionsupport program [7].

Access to all para clinical data have undeniable influence on a rapid and evidence-based decision making. Atkinson et al. have reported that EMRs support clinical decision-making; this information developed for medical clinics provide treatment suggestions based on the patient's signs, symptoms and clinical data. This system 
can be used to advise students and faculty of evidence-based clinical practice guidelines for various clinical situations and Electronic data interchange, including data sharing with other health professionals [8]. Williams et al. also showed the potential role of EMR systems on enhancement of healthcare delivery and facilitation of decision-making processes [3].

Another benefit of this project was the accessibility of information in each step and anywhere for those with defined access. In accordance to our result Sujansky reported that a computer database is useful because it allows and facilitates multiple parties in various locations to access the records concurrently [7]. In addition Lau et al. in a systematic review showed a 51\% chance that an EMR can improve physician's office practice, as well [9].

Patient Satisfaction was another benefit of this registration system. Liu et al. in a systematic review evaluated patient satisfaction after implementation of EMR and showed a positive patient satisfaction with EMR [5]. Another systematic review has found that electronic health records effects mostly neutral or positive on patient satisfaction [10]. On the other hand Likourezos et al. revealed that the EMR had no positive impact on patient care and satisfaction due to confusion in following the sequence of screens, the amount of time to use the EMR and the confidentiality of patient information [11].

We also found that EMR either in research program or for redirecting some problematic approaches could be beneficial. It will also provide an online Log Book for Fellows and Ob/Gyn residents working in this field. These systematic data can be exportable to other software packages for further analyses. Another report confirmed our results and indicated that a well-designed registry system can provide useful tool for both clinical and academic pursuits [6]. Diero et al. showed that using electronic medical records (EMRs) for prospective investigations and collecting outcomes data can increase the efficiency of such investigations [12].

Supervised data entry could guarantee the quality of information, with least of errors or neglects. Ornstein et al. showed the role of electronic medical record in quality of care improvement by providing point-of-care reminder, decision support tools and a database for substantiating the effectiveness of care [13].

At present we have multiple challenges that we hope to overcome:

- Extending the Registry system to ambulatory patients.

- Provision of off line data registry.

- Longer term follow up for at least 24 months.

- Validating and adding questionnaires like PFI-Q7, ...

For betterment of Female Pelvic Floor Health Program, a multidisciplinary approach including cooperation of Female Urology and Colorectal Groups in this project is needed.

For this matter and for country wide extension of the program we are trying in next steps to get Ministry of Health involved in this project. Also use of the data would be efficient in country wide female pelvic floor health policy making.

Naturally, in the transition period of changing the route of Data entry, there will be some resistances from the Female Pelvic Floor Dysfunction Fellows, Obstetrics and Gynecologists Residents. Although we hope that with becoming accustomed to the data entry and observing its undeniable profits especially easy access to Data, these reluctances will be overcome.

\section{Conclusion}

EMRs provide a registry system with the least possible missed data, evidence-based decision making, documented active patient follow up and increasing patient's satisfaction. This study should encourage others that EMRs can be used to improve the quality of care.

\section{References}

[1] Samoutis, G., Soteriades, E.S., Kounalakis, D.K., Zachariadou, T., Philalithis, A. and Lionis, C. (2007) Implementation of an Electronic Medical Record System in Previously Computer-Naive Primary Care Centres: A Pilot Study from Cyprus. Informatics in Primary Care, 5, 207-216.

[2] Lium, J.T., Tjora, A. and Faxvaag, A. (2008) No Paper, but the Sameroutines: A Qualitative Exploration of Experiences in Two Norwegian Hospitals Deprived of the Paper Based Medical Record. BMC Medical Informatics and Decision Making, 8, 2. http://dx.doi.org/10.1186/1472-6947-8-2

[3] Williams, F. and Boren, S. (2008) The Role of the Electronic Medical Record (EMR) in Care Delivery Development in 
Developing Countries: A Systematic Review. Informatics in Primary Care, 16, 139-145.

[4] Likourezos, A., Chalfin, D.B., Murphy, D.G., Sommer, B., Darcy, K. and Davidson, S.J. (2004) Physician and Nurse Satisfaction with an Electronic Medical Record System. Journal of Emergency Medicine, 27, 419-424. http://dx.doi.org/10.1016/j.jemermed.2004.03.019

[5] Liu, J., Luo, L., Zhang, R., Huang, T., Scand, J. and Caring, S. (2013) Patient Satisfaction with Electronic Medical/ Health Record: A Systematic Review. Scandinavian Journal of Caring Sciences, 27, 785-791. http://dx.doi.org/10.1111/scs.12015

[6] Roger, P., Goldberg, A., Gafni, K., Kelly J., Richard S., Darryck, M., et al. (2014) An Automatic Female Pelvic Medicine and Reconstructive Surgery Registry and Complications Manager Developed in an Electronic Medical Record. Female Pelvic Medicine \& Reconstructive Surgery, 20, 302-305. http://dx.doi.org/10.1097/SPV.0000000000000124

[7] Sujansky, W.V. (1998) The Benefits and Challenges of an Electronic Medical Record: Much More than a "WordProcessed" Patient Chart. WIM, 169, 176-184.

[8] Atkinson, J.C., Zeller, G. and Shah, C. (2002) Electronic Patient Records for Dental School Clinics: More than Paperless Systems. Journal of Dental Education, 66, 634-643.

[9] Lau, F., Price, M., Boyd, J., Partridge, C., Bell, H. and Raworth, R. (2012) Impact of Electronic Medical Record on Physician Practice in Office Settings: A Systematic Review. BMC Medical Informatics and Decision Making, 12, 10. http://dx.doi.org/10.1186/1472-6947-12-10

[10] Irani, J.S., Middleton, J.L., Marfatia, R., Omana, E.T. and D’Amico, F. (2009) The Use of Electronic Health Records in the Exam Room and Patient Satisfaction: A Systematic Review. Journal of the American Board of Family Medicine, 22, 553-562. http://dx.doi.org/10.3122/jabfm.2009.05.080259

[11] Likourezos, A., Chalfin, D.B., Murphy, D.G., Sommer, B., Darcy, K. and Davidson, S.J. (2004) Physician and Nurse Satisfaction with an Electronic Medical Record System. Journal of Emergency Medicine, 27, 419-424. http://dx.doi.org/10.1016/j.jemermed.2004.03.019

[12] Diero, L., Rotich, J.K., Bii, J., Mamlin, B.W., Einterz, R.M., Kalamai, I.Z. and Tierney, W.M. (2006) A ComputerBased Medical Record System and Personal Digital Assistants to Assess and Follow Patients with Respiratory Tract Infections Visiting a Rural Kenyan Health Centre. BMC Medical Informatics and Decision Making, 6, 21. http://dx.doi.org/10.1186/1472-6947-6-21

[13] Ornstein, S.M., Oates, R.B. and Fox, G.N. (1992) The Computer-Based Medical Record: Current Status. Journal of Family Practice, 35, 556-565. 